\title{
LIBS Monitoring and Analysis of Laser-Based Layered Controlled Paint Removal from Aircraft Skin
}

\author{
Wenfeng Yang $D^{1},{ }^{1}$ Ziran Qian, ${ }^{1}$ Yu Cao, ${ }^{2}$ YongChao Wei, ${ }^{3}$ Chanyuan Fu, ${ }^{1}$ TianQuan Li, \\ Dehui Lin, ${ }^{1}$ and Shaolong $\operatorname{Li}^{1}{ }^{1}$ \\ ${ }^{1}$ Aviation Engineering Institute, Civil Aviation Flight University of China, Guanghan 618307, China \\ ${ }^{2}$ Institute of Laser and Optoelectronic Intelligent Manufacturing, Wenzhou University, Wenzhou 320000, China \\ ${ }^{3}$ Department of Research, Civil Aviation Flight University of China, Guanghan 618307, China
}

Correspondence should be addressed to Wenfeng Yang; ywfcyy@163.com and Shaolong Li; zlishaolong@163.com

Received 3 July 2021; Revised 17 August 2021; Accepted 4 September 2021; Published 30 September 2021

Academic Editor: Nik a Krstulovi

Copyright $(92021$ Wenfeng Yang et al. This is an open access article distributed under the Creative Commons Attribution License, which permits unrestricted use, distribution, and reproduction in any medium, provided the original work is properly cited.

Reliability and controllability of selective removal of multiple paint layers from the surface of aircraft skin depend on effective online monitoring technology. An analysis was performed on the multi-pulse laser-induced breakdown spectroscopy (LIBS) on the surface of the aluminum alloy substrate, primer, and topcoat. Based on that, an exploration was conducted on the changes of the characteristic peaks corresponding to the characteristic elements that are contained in the topcoat, primer, and substrate with different layers of a laser action, in combination with analysis of microscopic morphology, composition, and depth of laser multipulse pits. The results show that the appearance and increase of the characteristic peak intensity of the Ca I at the wavelength of $422.7 \mathrm{~nm}$ can be regarded as the basis for the complete removal of the topcoat; the decrease or disappearance of the characteristic peak intensity can be regarded as the basis for the complete removal of the primer. Al I spectrum at the wavelength of $394.5 \mathrm{~nm}$ and $396.2 \mathrm{~nm}$ can be adopted to characterize the degree of damage to the aluminum alloy substrate. The feasibility and accuracy of the LIBS technology for the laser selective paint removal process and effect monitoring of aircraft skin were verified. Demonstrating that under the premise of not damaging the substrate, laser-based layered controlled paint removal (LLCPR) from aircraft skin can be achieved by monitoring the spectrum and composition change law of specified wavelength position corresponding tothe characteristic elements that are contained in the specific paint layer.

\section{Introduction}

The surface coating of aircraft skin is a complex multi-layer structure, which could realize its functionality and decoration. However, aircraft will inevitably be affected by natural erosion and chemical corrosion during its operation and service, which would result in aging, cracking, and peeling of the paint on the skin surface.

To ensure safe and reliable operation of the aircraft, overall or partial paint removal from the skin and paint spraying are required to restore its surface coating during repair, maintenance, passenger and cargo aircraft modification, or carrier replacement. Such processes shall be conducted according to the layered controlled paint removal (LCPR) or thickness controlled paint removal (TCPR) requirements of the Aircraft Maintenance Manual (AMM) [1].
Currently, there are some deficiencies in common chemical methods, manual polishing, and shot-peening processes applied in engineering, such as serious pollution, damage to health, and considerable expenses [2]. Besides, it is cumbersome and uncontrollable for the combined method of thickness measurement statistics and engineering experience to judge the paint removal process, which is limited by high consumption and low efficiency. It is difficult to satisfy the demand for online monitoring and feedback of the paint removal process and effect in quality control of paint removal from aircraft skin.

Recently, laser paint removal has received widespread attention due to its high efficiency, high precision, "green," intelligence, and controllability. This technology refers to a technique of irradiating a high-energy laser beam to the part of the paint to be removed, using the ablation, vibration, and 
plasma effect of the paint and the substrate to remove the paint on the surface of the material [3]. This technology has been widely used in steel [4], cars [5], nuclear power [6], and other fields, and it is expected to partially or completely replace traditional removal methods in the future.

Laser-induced breakdown spectroscopy (LIBS) is cutting-edge technology in the field of online monitoring and feedback. The principle is to concentrate the laser pulse with high energy density on the surface of the sample to be tested, and plasma would be excited on the surface. And through analyzing the characteristic spectral lines radiated by the electron energy level transition of the particles in the plasma, qualitative or quantitative information of the elements that are contained in the sample can be obtained. This technology is characterized by efficiency and accuracy [7]. Nowadays, there are abundant LIBS systems that have been maturely applied in Mars exploration [8, 9], medicine [10], food [11], environmental monitoring [12], cultural relics and works of art [13], metal recycling [14], and soil composition analysis [15].

Based on the correlation between laser paint removal and the principle of LIBS, LIBS technology can be integrated into the laser paint removal process by the plasma generated in this process [16]. The plasma spectrum of the paint removal process can be collected online to determine the paint removal effect. According to the paint removal effect, the cleaning conditions would be modulated during the cleaning process in an attempt to achieve intelligent control. Such modulation could reduce the risk of damage to the structure and performance of the substrate resulted from mismatched parameter settings. And it is expectable to realize automatic monitoring and optimized control of laser cleaning [17].

Currently, scholars have conducted many studies on LIBS online monitoring and feedback of the laser cleaning process. Xing Li et al. applied LIBS for real-time monitoring of laser cleaning hard oxide layer for hot-rolled stainless steel, the results show that the relative intensity ratio (RIR) of Fe I emission line at $520.9 \mathrm{~nm}$ and Cr I emission line at 589.2 could be a quantitative index to monitor the cleaning process. When the RIR sharply decreases at a local region of the surface to be cleaned, the power of the laser should be increased to ensure that the oxide layer is effectively removed [18]. Lanxiang Sun et al. used LIBS technology designed laser online monitoring system for cleaning quality of carbon fiber composite; the $\mathrm{Na}$ I characteristic spectral lines at $588.955 \mathrm{~nm}$ and $589.414 \mathrm{~nm}$ were selected; and the Pearson correlation coefficient analysis method was used to online evaluate the laser cleaning effect [19]. Yanqun Tong et al. have also researched the LIBS spectrum online detection of laser cleaning carbon fiber composite. S II spectrum with the wavelength of $393.3 \mathrm{~nm}$ and $589.5 \mathrm{~nm}$ can effectively characterize the surface cleaning quality online [20]. In addition, Yanqun Tong et al. also studied the relationship between the LIBS characteristic line and the pulse numbers during the laser cleaning process of aluminum alloy before welding and proposed the $\mathrm{O} / \mathrm{Al}$ characteristic line intensity ratio as the basis for the online detection of cleaning effect and secondary oxidation damage. And X-ray photoelectron spectroscopy analysis was used to verify the accuracy of the judgment basis [21]. In terms of laser paint removal, based on LIBS technology, Kangxi Chen et al. online measured the LIBS spectrum of the paint surface under different nanosecond laser energy, analyzed the paint ablation morphology by scanning electron microscope (SEM), and used an X-ray energy spectrometer to measure the changes of element composition before and after laser paint removal. Confirming the ablation effect of the laser paint removal process [22]. Lin Chen et al. measured the spectral signal of plasma during the laser removal of the single-layer paint on the aluminum alloy surface. They investigated the evolution of the spectral characteristic peak intensity of characteristic elements in the paint over time [23]. Qionghua Zhou et al. fitted the time-resolved characteristics of LIBS characteristic peaks. Their finding indicates that it was feasible to monitor the laser paint removal process by monitoring the sudden change of the ratio of the background spectrum to the characteristic spectrum of the characteristic peak at a fixed time (such as $0.3 \mu \mathrm{s}$ ) [24]. The above research proves the feasibility of online monitoring of laser cleaning quality with LIBS technology. However, there were few endeavors to explore the monitoring method of laser-based layered controlled paint removal (LLCPR) and laser-based thickness controlled paint removal (LTCPR) of complex multi-layer paint structure on the surface of the aircraft skin.

In this work, we researched the single-pulse removal process of single-layer paint and two-layer paint on the surface of the aluminum alloy aircraft skin. The multi-pulse continuous LIBS signals were employed to determine the characteristic elements and corresponding characteristic peaks of a specific layer. And the changes of the characteristic peaks of LIBS with the specific paint layer and pulse numbers were explored. Moreover, the LIBS online monitoring method for laser-based layered controlled paint removal (LLCPR) from aluminum alloy aircraft skin was analyzed.

\section{Experimental Section}

2.1. Experimental Device. Leica's DM6 M LIBS device was utilized in this work. The resolution of DM6 M optical microscope was $2.35 \mu \mathrm{m} \times 2.35 \mu \mathrm{m}$, and $Z$-axis accuracy was $0.3 \pm 0.1 \mu \mathrm{m}$. LIBS system was composed of a pulsed nitrogen laser and a CCD spectroscope. The laser wavelength was $337.1 \mathrm{~nm}$; pulse energy was $90 \mu \mathrm{J}$; repetition rate was $1 \mathrm{~Hz}$; pulse width was $3 \mathrm{~ns}$; spot diameter was $15 \mu \mathrm{m}$ at the focal point; and collection spectrum wavelength range was $360 \mathrm{~nm}-700 \mathrm{~nm}$. During the test process, the laser beam passed through a focusing lens with a focal length of $120 \mathrm{~mm}$ to make the energy focused on the sample surface; the plasma radiation spectrum generated by the laser was coupled to a spectrometer (Andor, Me5000) through a fiber probe for spectrum collection; the system software (LAS X) automatically preprocessed the collected raw spectral data such as normalization and noise reduction. The laser and the spectrometer were synchronously controlled by a digital delay generator (Berkeley Nucleonics Corp, BNC575) and triggered by the $Q$-switched signal of the nanosecond laser. 
To avoid excessive laser action on the sample surface, the sample was placed on a three-dimensional translation stage so that each laser pulse could act on a new position of the sample. A schematic diagram of the experimental device is shown in Figure 1.

2.2. Experimental Sample. 2024-T3 Aluminum alloy commonly used for aircraft skin was adopted. Original aluminum alloy surface was pretreated by degreasing, decontamination, and polishing and then uniformly sprayed with yellow epoxy primer in the thickness of about $30 \mu \mathrm{m}$ and white polyurethane topcoat in the thickness of about $60 \mu \mathrm{m}$. Figure 2 shows the observation results of the crosssection of a two-layer spray paint sample with the assistance of a trinocular continuous zoom stereo microscope. The aluminum alloy substrate, yellow primer, and white topcoat could be observed. The thickness of the primer is about $30.10 \mu \mathrm{m}$, and that of the topcoat is about $60.15 \mu \mathrm{m}$.

\section{Multi-Pulse LIBS Analysis of Aluminum Alloy Substrate}

3.1. Characteristic Peaks of Multi-Pulse LIBS Spectrum of Aluminum Alloy Substrate. The laser was focused on the surface of the sample to be tested, and the surface of the material absorbed the laser energy to generate plasma. During the plasma cooling process, photons of a specific frequency, namely the characteristic spectral lines of the element, were generated. Figure 3 shows the $360 \mathrm{~nm}-700 \mathrm{~nm}$ LIBS pretreatment spectra after the first pulseapplied, vertically to the surface of the aluminum alloy substrate.

According to the Atomic Spectra Database (ASD) of the National Institute of Standards and Technology (NIST), the main characteristic spectral lines at the first pulse were calibrated, as listed in Table 1. The characteristic peaks of $\mathrm{Mn}$ I, Al I, Cu I, Cr I, Zn I, Mg I, and Si I were detected. The spectra and their intensities look the same for subsequent pulses, such as the third and fifth pulses. By drawing a comparison with the database standard spectrum, there were errors in the measured correlation spectrum, which may be caused by environmental factors and matrix effects. The cause of the matrix effects is the difference in the physical and chemical properties of the sample, as well as the nonlinearity of the interaction between the laser and the substance. This nonlinearity causes the electron temperature, electron density, and ablation amount to be affected during laser ablation, plasma generation, and expansion $[25,26]$.

\subsection{Multi-Pulse LIBS Pits in Aluminum Alloy Substrate.} The depth of the pit formed by the laser along the incident direction on the sample surface was defined as the laser action depth. The DM6 M optical microscope was adopted, and the depth of field synthesis method was employed to measure the action depth of the first, third, and fifth laser pulses. The measured depth was about $2.30 \mu \mathrm{m}, 6.56 \mu \mathrm{m}$, and $7.17 \mu \mathrm{m}$, respectively. Indicating that the cumulative laser energy absorbed by the aluminum alloy increased with the increase of pulse numbers, which caused a more intense

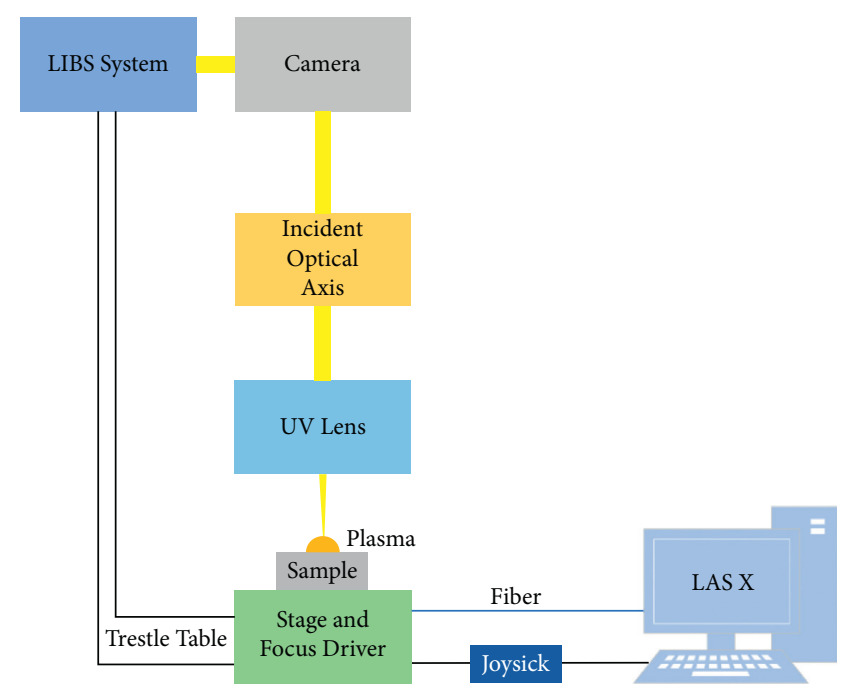

FIGURE 1: Experimental setup for DM6 M laser-induced breakdown spectroscopy.

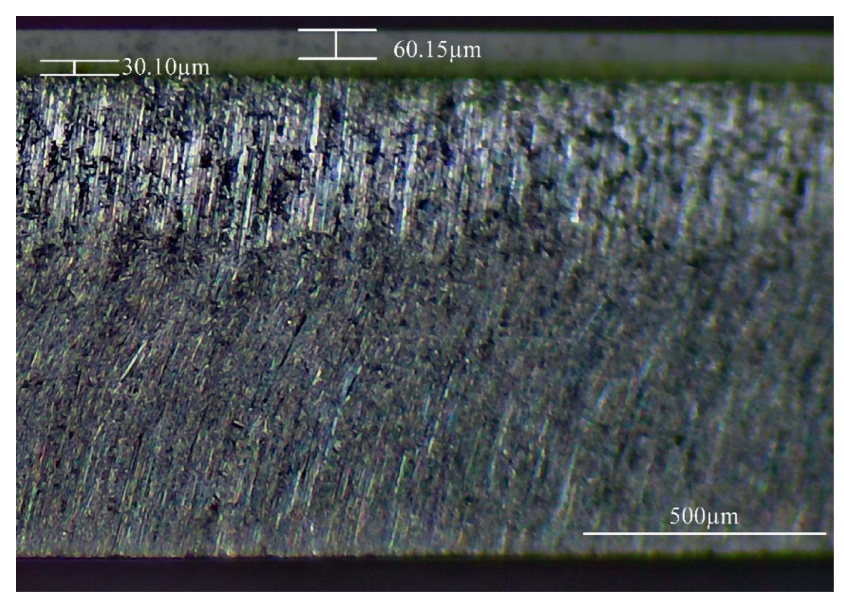

FIGURE 2: Aluminum alloy sample sprayed with yellow primer $(30 \mu \mathrm{m})$ and white topcoat $(60 \mu \mathrm{m})$.

physical and chemical reaction between the laser and aluminum alloy. As a result, the laser action depth increased.

Pits on the surface of the aluminum alloy substrate after the fifth laser pulse was selected for microscopic morphology and composition research. The test results of scanning electron microscope (SEM) and energy-dispersive spectroscopy (EDS) are shown in Figures 4(a) and 4(b), respectively. Combined with the EDS results of the original aluminum alloy, shown in Figure 4(d), comparing the composition test results of EDS and LIBS.

In Figure 4(a), it can be observed that the substrate under the fifth laser pulse has been completely ablated and was in a molten state. The reason was that the surface temperature of the substrate in the pit area during the laser action reached the lowest melting temperature of aluminum alloy $\left(660.37^{\circ} \mathrm{C}\right)$. The EDS test results indicated that the composition of the pit on the surface of the aluminum alloy substrate after the fifth laser pulse was consistent with the original aluminum alloy. However, the content of the $\mathrm{Zn}$ element increased, while that 


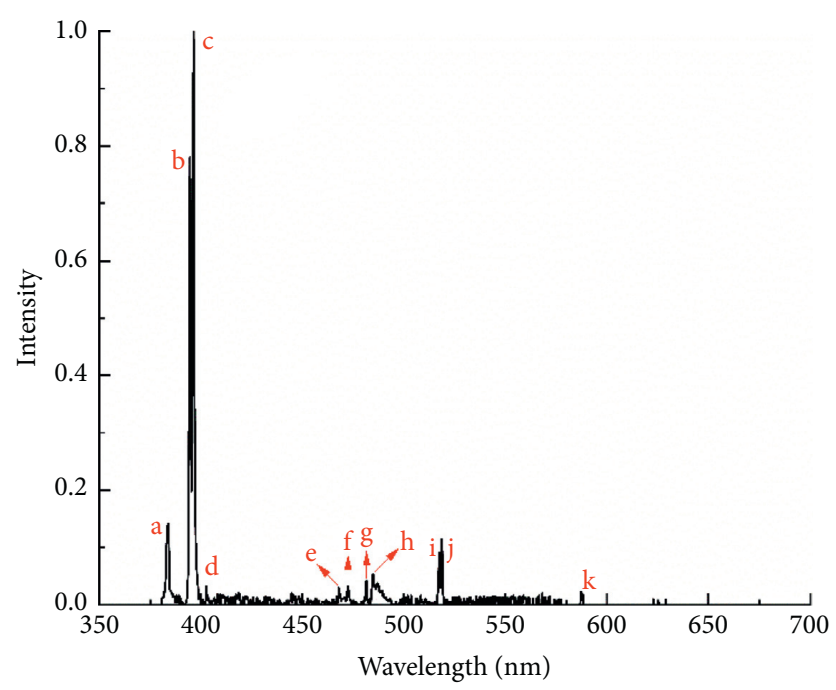

FIGURE 3: Laser-induced breakdown spectroscopy signal of aluminum alloy substrate after the first pulse acting vertically on the surface.

Table 1: Main characteristic spectral lines of 2024 aluminum.

\begin{tabular}{|c|c|c|c|c|}
\hline Serial number & Characteristic spectral line (nm) & Relevant spectral lines $(\mathrm{nm})$ & Atom & Error value $(\mathrm{nm})$ \\
\hline $\bar{a}$ & 383.4360 & 383.5 & Mn I & 0.0640 \\
\hline $\mathrm{b}$ & 394.4006 & 394.5 & $\mathrm{Al} \mathrm{I}$ & 0.1994 \\
\hline c & 396.1520 & 396.2 & $\mathrm{Al} \mathrm{I}$ & 0.3480 \\
\hline $\mathrm{d}$ & 402.7026 & 402.7 & $\mathrm{Cu} \mathrm{I}$ & 0.0026 \\
\hline $\mathrm{e}$ & 467.7340 & 467.8 & $\mathrm{Cu} \mathrm{I}$ & 0.0660 \\
\hline $\mathrm{f}$ & 472.3097 & 472.3 & Cr I & 0.0097 \\
\hline $\mathrm{g}$ & 481.0532 & 481.1 & $\mathrm{Zn} \mathrm{I}$ & 0.0468 \\
\hline $\mathrm{h}$ & 484.2290 & 484.2 & $\mathrm{Cu} \mathrm{I}$ & 0.0290 \\
\hline $\mathrm{i}$ & 517.2684 & 517.2 & Mg I & 0.0684 \\
\hline j & 518.3604 & 518.4 & Mg I & 0.0396 \\
\hline $\mathrm{k}$ & 587.3764 & 587.3 & Si I & 0.0764 \\
\hline
\end{tabular}

of the Mg element decreased. Such difference may be caused by the surface temperature of aluminum alloy instantaneously reached the rising temperature of $\mathrm{Zn}\left(907.00^{\circ} \mathrm{C}\right)$ when the high-energy laser interacted with the aluminum alloy and then deposited in the pit. Figures 5(a) and 5(b) show the stratified images of the $\mathrm{Zn}$ element of EDS of the original aluminum alloy and the pit; the concentration of $\mathrm{Zn}$ in the pit increased. In addition, due to the high-temperature conditions, $\mathrm{Mg}$ would react with various gaseous substances, such as $\mathrm{O}_{2}, \mathrm{~N}_{2}$, and $\mathrm{CO}_{2}$, and it may be inconsistency in the elemental composition of the crystal grains that formed the solid substance. The crystal grains that were utilized to make up the pit may not contain $\mathrm{Mg}$ elements, as shown in the circular area in Figure 5(d), which induced a decrease in the content of the Mg element.

EDS and LIBS detected the same elemental composition, which demonstrated the feasibility of LIBS for the analysis of sample surface composition.

Since the content of the $\mathrm{Al}$ element is the highest and the peak intensity of the sensitive spectral line reached the maximum value, Al I spectral line at $394.5 \mathrm{~nm}$ and $396.2 \mathrm{~nm}$ could be selected as a parameter for evaluating whether the laser has acted on the aluminum alloy.

\section{Multi-Pulse LIBS Analysis of Paint}

4.1. Spectral Characteristic Peaks of Epoxy Primer. To determine the correlation between the LIBS signal and laser primer removal, an analysis was performed on the changes in the LIBS signal of different pulse numbers along the primer surface.

Figure 6(a) shows the LIBS spectrum of the first pulse acting vertically on the primer surface. Multiple peaks of Ti, $\mathrm{Ca}$, and $\mathrm{Zn}$ were detected, and the corresponding wavelength positions are summarized in Table 2. The characteristic peak intensity of $\mathrm{Ti}$ I reached the maximum value at $453.6 \mathrm{~nm}$.

Figures 6(b)-6(f) show the LIBS signals when the pulse number was $3,5,10,20$, and 23 , respectively. The characteristic peaks were the same as those in Figure 6(a), and the average line intensity of the Ti element was greater than that of the $\mathrm{Ca}$ and $\mathrm{Zn}$ elements. It can be demonstrated that the component elements of the primer contained $\mathrm{Ti}, \mathrm{Ca}, \mathrm{Zn}$, and the content of Ti may be greater than that of $\mathrm{Ca}$ and $\mathrm{Zn}$.

When the pulse number was 33, the characteristic peak of Mn I (383.5 nm), Al I (394.5 nm and $396.2 \mathrm{~nm}), \mathrm{Cu} \mathrm{I}$ $(484.2 \mathrm{~nm})$, and $\mathrm{Mg} \mathrm{I}(517.2 \mathrm{~nm}$ and $518.4 \mathrm{~nm})$ appeared, 


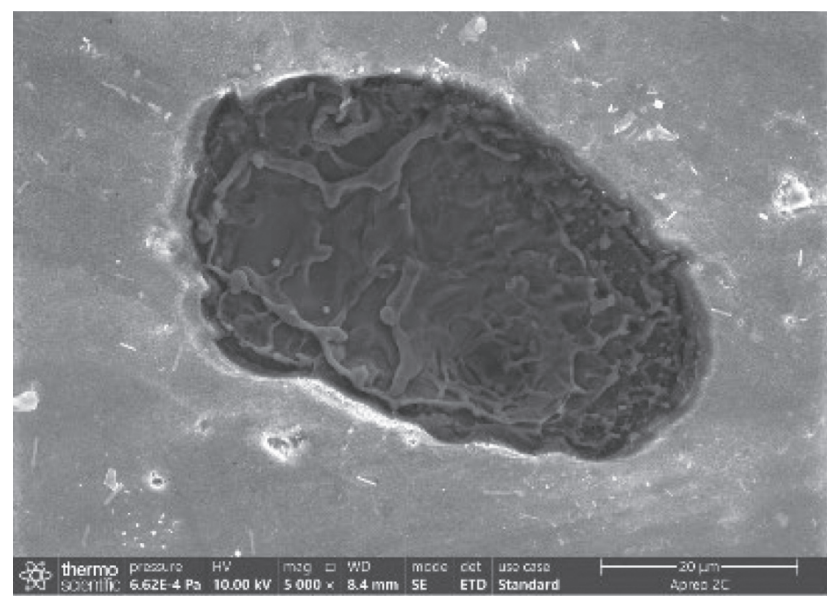

(a)

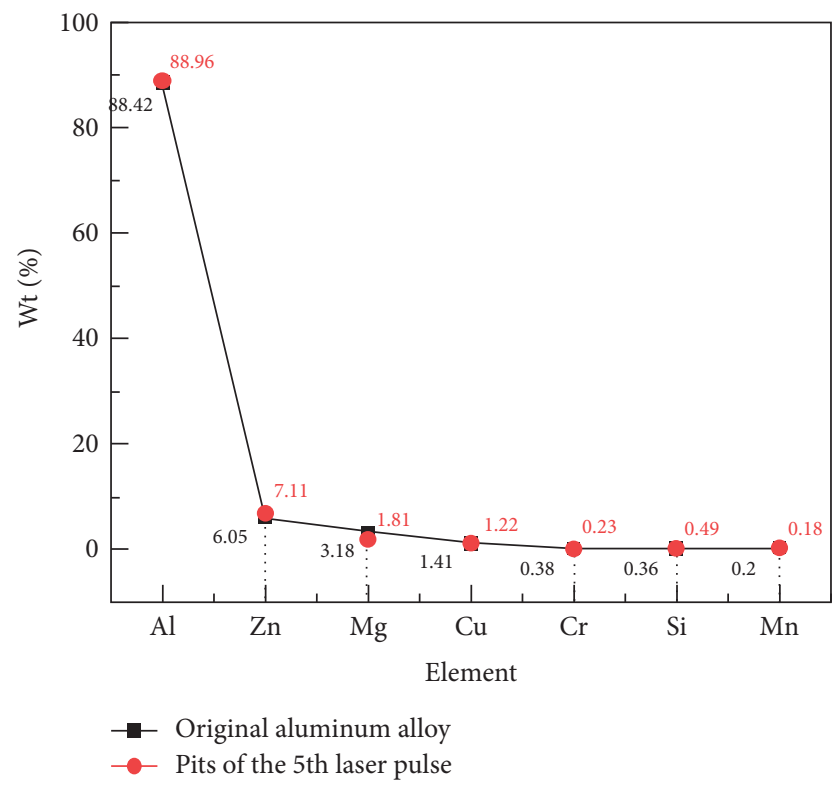

(c)

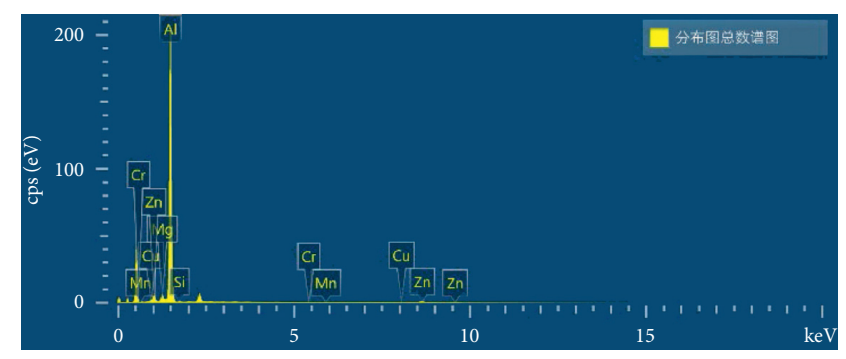

(b)

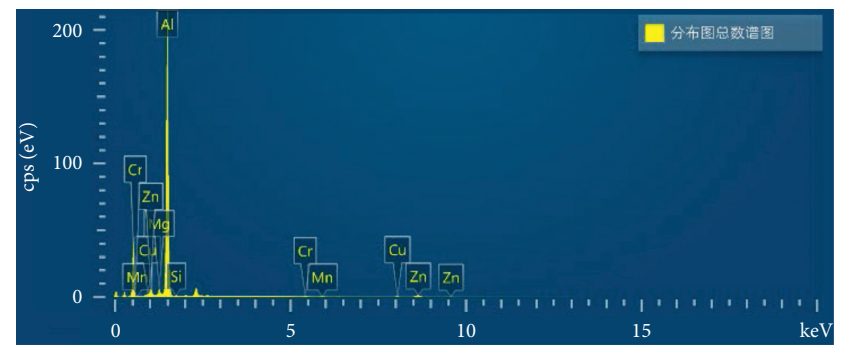

FIGURE 4: Pit morphology and EDS of aluminum alloy: (a) SEM, (b) EDS of the pit after the action of the fifth laser pulse, (c) EDS test results, and (d) EDS of raw aluminum alloy.

while that of $\mathrm{Zn} \mathrm{I}$ at $468.0 \mathrm{~nm}$ and $\mathrm{Ti}$ and $\mathrm{Ca}$ elements all disappeared, as shown in Figure 6(g). Comparing the characteristic peak of aluminum alloy in Figure 3 and Table 1 , it could be observed that the laser acted on the surface of the aluminum alloy at this pulse. The changes in the characteristic peak of $\mathrm{Zn}$ indicated that under the same conditions, there was a correlation between the position of the plasma spectrum line excited by the material after absorbing the laser energy and the material properties.

When the pulse number of pulses was 36, the $\mathrm{Cr}$ $(472.3 \mathrm{~nm})$ element with a lower content $(0.10 \%)$ in the aluminum alloy appeared, as shown in Figure 6(h).

In addition, an analysis was performed on the microscopic morphology of the pit after the 36th pulse. Under an optical microscope, metallic luster and molten aluminum alloy can be observed at the bottom of the ablation pit, as shown in Figure 7(a). The laser action depth measured by the combined depth of field measurementwas about $42.61 \mu \mathrm{m}$, as shown in Figure 7(b), which was greater than the thickness $30 \mu \mathrm{m}$ of the primer. Hence, it could be demonstrated that the laser acted on the aluminum alloy substrate at this pulse.

The laser scanning confocal microscope (LSCM) function of the 3D profiler (Sneox 090) was adopted to scan the interface between the pit and the original paint surface. The difference between the average height on both sides of the interface was calculated by 10 repeated measurements. And the average action depth of 36 pulses was about $42.5602 \mu \mathrm{m}$, as shown in Figure $8(\mathrm{~d})$. The standard deviation of the measured value was 0.7709 . Figures $8(\mathrm{a})-8(\mathrm{c})$ show the results of one of the ten measurements. Figure $8(\mathrm{c})$ is a schematic diagram of the cross-section that was plotted based on the height of the dashed line in Figure 8(a); the test result was $42.5090 \mu \mathrm{m}$. Demonstrating the depth of field measurement is accurate. 


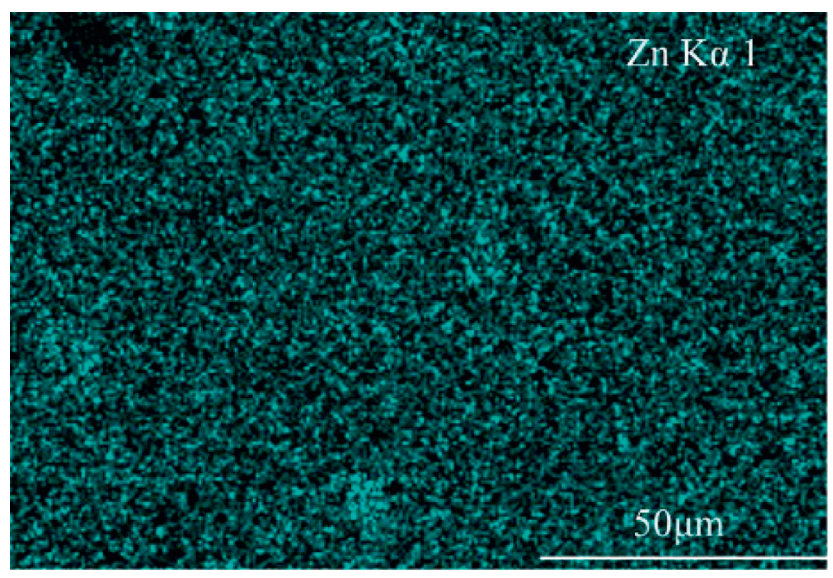

(a)

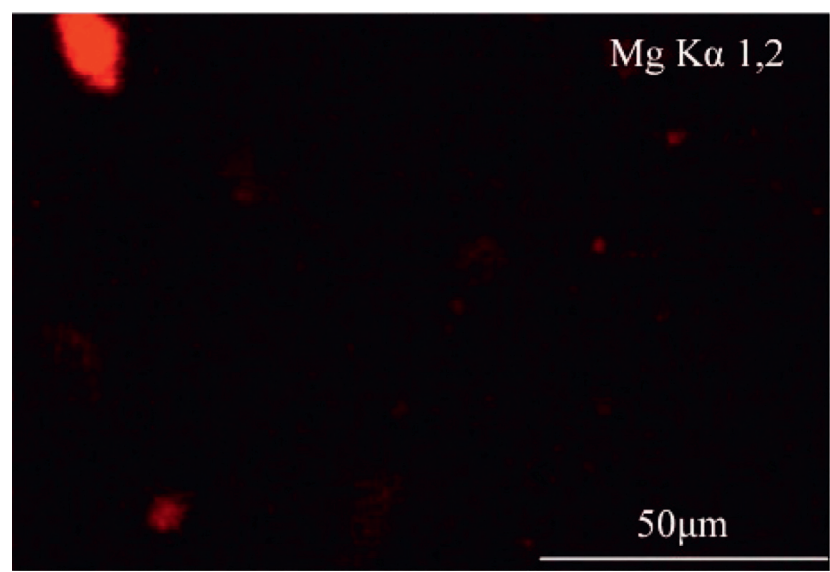

(c)

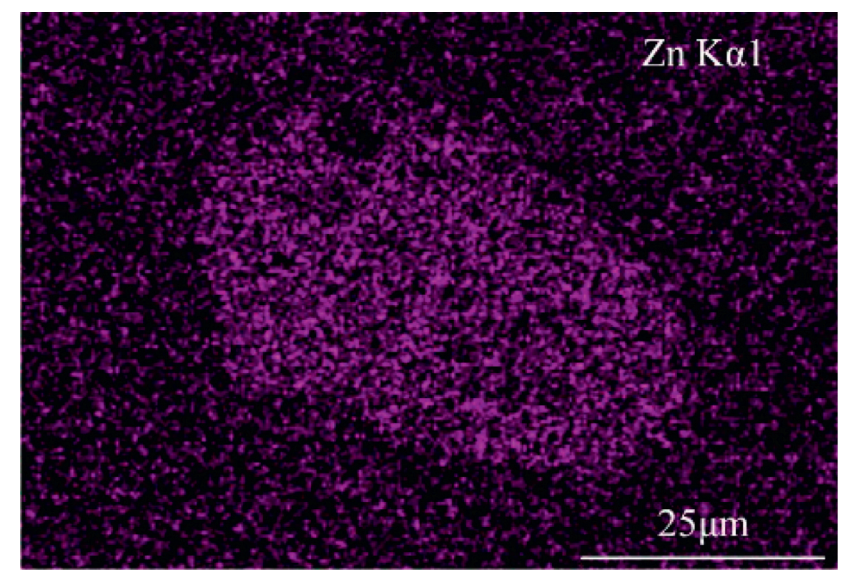

(b)

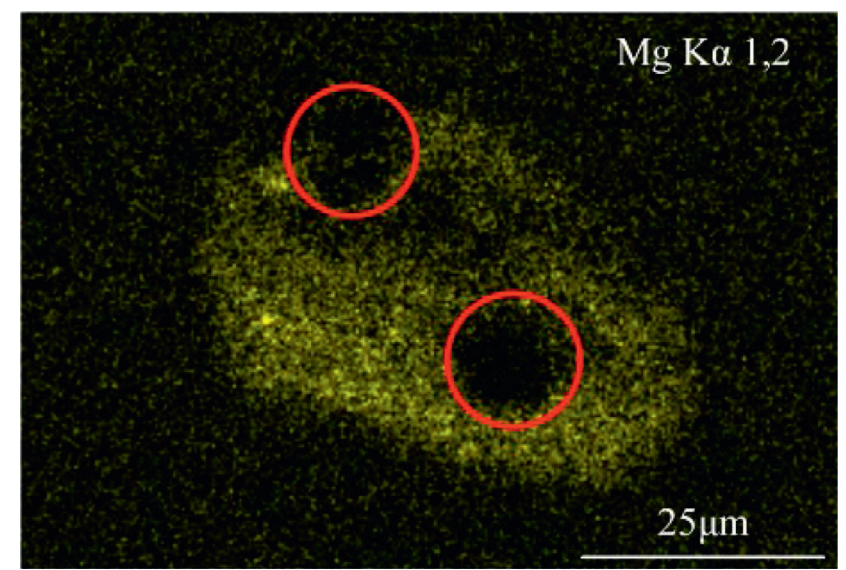

(d)

Figure 5: Stratified image of EDS: (a) Zn, (b) Zn, (c) Mg of the original aluminum alloy, and (d) Mg of the pit after the action of the fifth laser pulse.

According to the changes of the characteristic peaks of the elements at the 33rd pulse, the characteristic peaks of $\mathrm{Mn}, \mathrm{Al}, \mathrm{Zn}, \mathrm{Cu}, \mathrm{Ti}$, and $\mathrm{Ca}$ could be selected to monitor the primer removal process. The disappearance of characteristic peaks of $\mathrm{Ti}, \mathrm{Ca}$, and $\mathrm{Zn}$ elements or the appearance of characteristic peaks of $\mathrm{Mn}, \mathrm{Al}$, and $\mathrm{Cu}$ could demonstrate the removal of the primer. Besides, the atomic states characteristic peaksTi and $\mathrm{Ca}$ were most sensitive at $453.6 \mathrm{~nm}$ and $422.7 \mathrm{~nm}$, respectively. The content of the $\mathrm{Zn}$ element that served the pigment and filler of the paint is low, with the characteristic peak being insensitive and similar to that of the substrate characteristic peak at $481.1 \mathrm{~nm}$; the contents of $\mathrm{Mn}$ and $\mathrm{Cu}$ elements in 2024 aluminum alloy are low, and the characteristic peaks were not obvious; the appearance of high-strength characteristic peaks of $\mathrm{Al}$ element proves that the substrate has been damaged. Based on the aforementioned facts, the spectral line intensity of Ca I at $422.7 \mathrm{~nm}$ and Ti I at $453.6 \mathrm{~nm}$ were selected as the parameter for evaluating whether the primer could be completely removed.

Figure 9 shows the changes of the characteristic peak intensity of Al I at $394.5 \mathrm{~nm}$ and $396.2 \mathrm{~nm}$, Ca II at $393.4 \mathrm{~nm}$, and $\mathrm{Ca} \mathrm{I}$ at $422.7 \mathrm{~nm}$ during the primer removal process. It can be observed that with the increase of the pulse numbers, the characteristic peak intensity of the Ca element increased first and then disappeared suddenly when the characteristic peak of the Al element suddenly increased. It could be demonstrated that with the continuation of the action process of the laser and the primer, the characteristic peaks of the characteristic elements of the primer became increasingly obvious. When the laser was applied to the substrate, the characteristic peaks of the sensitive spectral lines of the characteristic elements of the primer disappeared immediately, and the characteristic peaks of the sensitive spectral lines of the sensitive elements in the substrate characteristic elements appeared immediately.

4.2. Spectral Characteristic Peaks of Polyurethane Topcoat. To determine the corresponding relationship between the LIBS signal and the process of laser remove topcoat and the primer removal, we analyzed the LIBS signal changes at different pulse numbers along the surface of the topcoat.

Figure 10(a) shows the LIBS spectrum of the first pulse acting vertically on the surface of the topcoat. The same Ti I peak as the primer and the $\mathrm{Na}$ I peak at $588.9 \mathrm{~nm}$ 


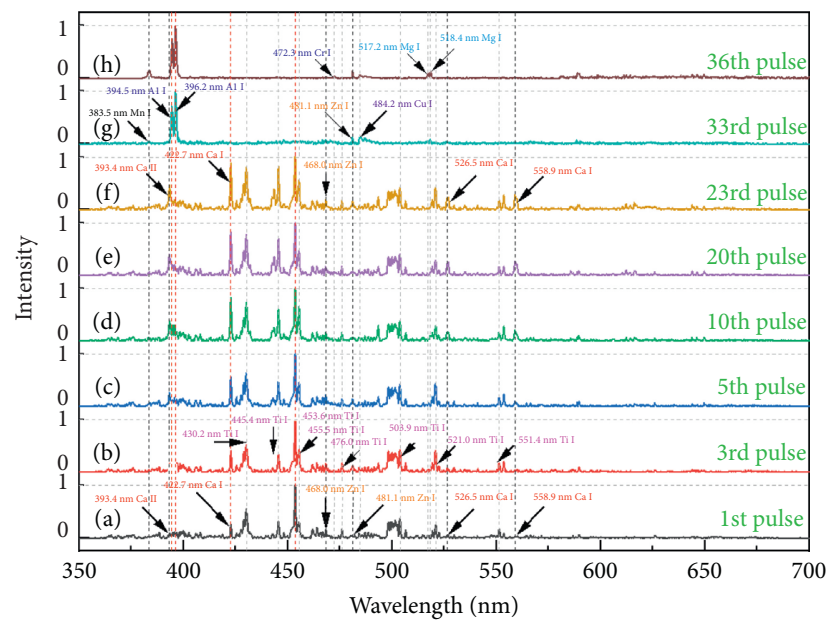

FIGURE 6: Laser-induced breakdown spectroscopy signal of primer after different times of laser pulse acting vertically on the surface: (a) 1 , (b) 3, (c) 5, (d) 10, (e) 20, (f) 23, (g) 33, and (h) 36 .

TABLE 2: Main characteristic spectral lines of yellow epoxy primer.

\begin{tabular}{lccc}
\hline Characteristic spectral line $(\mathrm{nm})$ & Relevant spectral lines $(\mathrm{nm})$ & Atom & Error value $(\mathrm{nm})$ \\
\hline 430.1079 & 430.2 & Ti I & 0.0921 \\
445.3851 & 445.4 & Ti I & 0.0149 \\
453.6040 & 453.6 & Ti I & -0.0040 \\
455.5069 & 455.5 & Ti I & -0.0069 \\
475.9269 & 476.0 & Ti I & 0.0731 \\
503.8397 & 503.9 & Ti I & 0.0603 \\
521.0384 & 521.0 & Ti I & -0.0384 \\
551.4343 & 551.4 & Ti I & -0.0343 \\
393.3660 & 393.4 & Ca II & 0.0340 \\
422.6730 & 422.7 & Ca I & 0.0270 \\
526.5560 & 526.5 & Ca I & -0.0560 \\
558.8760 & 558.9 & Ca I & 0.0240 \\
468.0136 & 468.0 & Zn I & -0.0136 \\
481.0532 & 481.1 & Zn I & 0.0468 \\
\hline
\end{tabular}

$(588.9951 \mathrm{~nm})$ were detected. It could be demonstrated that $\mathrm{Ti}$ and $\mathrm{Na}$ were the surface elements of the white polyurethane topcoat, and the content of the Ti element was high in the topcoat and primer.

After the 10th, 20th, 30th, and 39th pulses were acted vertically on the topcoat, the LIBS spectrum remained unchanged, as shown in Figures 10(b)-10(e), respectively. It means that the laser was still acting on the topcoat.

The depth of laser action was continued to increase. When the pulse number reached 40 , the characteristic peaks of Ca II $(393.4 \mathrm{~nm})$, Ca I $(422.7 \mathrm{~nm})$, and Zn I (468.0 nm, $481.1 \mathrm{~nm})$ appeared, and the characteristic peaks of $\mathrm{Na}(588.9 \mathrm{~nm})$ disappeared, as shown in Figure 10(f). Compared with Figure 6, it can be observed that the laser may act on the primer at this pulse. Therefore, the spectral intensity of Ca II at $393.4 \mathrm{~nm}$, Ca I at $422.7 \mathrm{~nm}, 468.0 \mathrm{~nm}, \mathrm{Zn} \mathrm{I}$ at $481.1 \mathrm{~nm}$, and $\mathrm{Na} \mathrm{I}$ at $588.9 \mathrm{~nm}$ can be selected as the parameter for evaluating whether topcoat has been completely removed.

When the pulse numberwas 45, 62, and 64, respectively, the LIBS spectrum was the same as Figure 10(f), as shown in Figures $10(\mathrm{~g})-10(\mathrm{i})$.
When the pulse number was 65, the $\mathrm{Al}$ element at $394.5 \mathrm{~nm}$ and $396.2 \mathrm{~nm}$ appeared; the characteristic peaks of $\mathrm{Ca}$ and $\mathrm{Zn}$ disappeared; and the characteristic peaks of $\mathrm{Ti}$ remained unchanged, as shown in Figure 10(j). It could be demonstrated that the laser had just acted on the aluminum alloy substrate, and the $\mathrm{Al}$ element was sensitive to the appearance of the aluminum alloy substrate. Therefore, the spectral intensity of Ca II at $393.4 \mathrm{~nm}$, Ca I at $422.7 \mathrm{~nm}, \mathrm{Zn} \mathrm{I}$ at $468.0 \mathrm{~nm}$ or $481.1 \mathrm{~nm}$, and Al I at $394.5 \mathrm{~nm}$ or $396.2 \mathrm{~nm}$ can be selected as the parameters for evaluating whether the primer has been completely removed after the removal of the topcoat.

As per the above analysis, different paint layers of the laser cleaning corresponded to different LIBS characteristic peaks. The existence of LIBS characteristic peak of Na element demonstrated that the laser was cleaning the topcoat. The increase of the LIBS characteristic peak of the Ca element demonstrated that the laser started to clean the primer. And when the LIBS high-intensity characteristic peak of the Al element appears, it indicates that the laser starts to damage the substrate. 


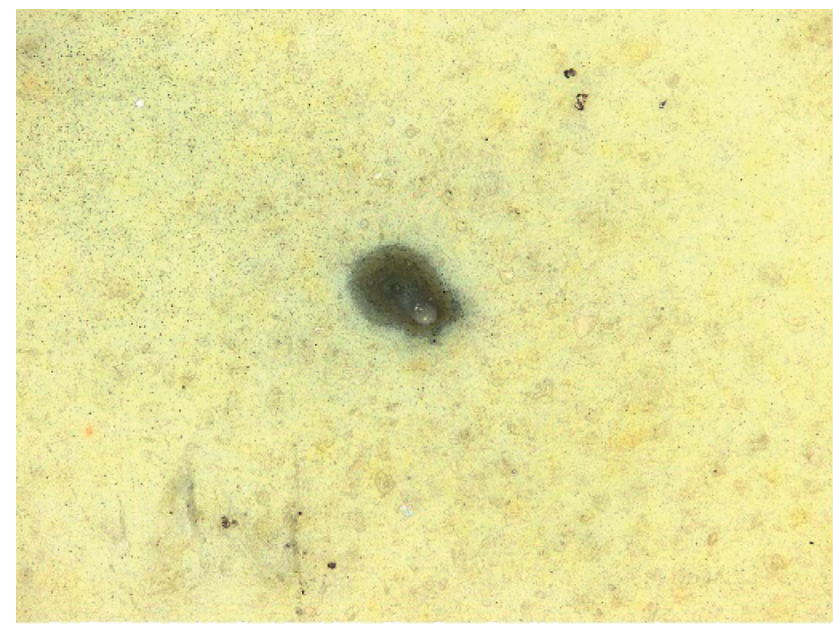

(a)

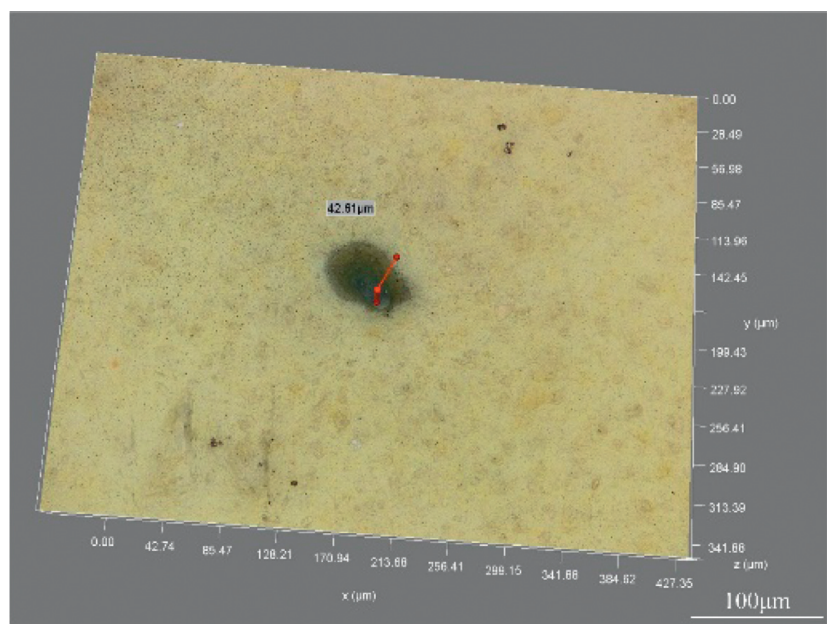

(b)

Figure 7: Measurement of the surface microscopic morphology and depth after 36 laser pulses vertical action primer surface: (a) microscopic morphology and (b) depth measurement.

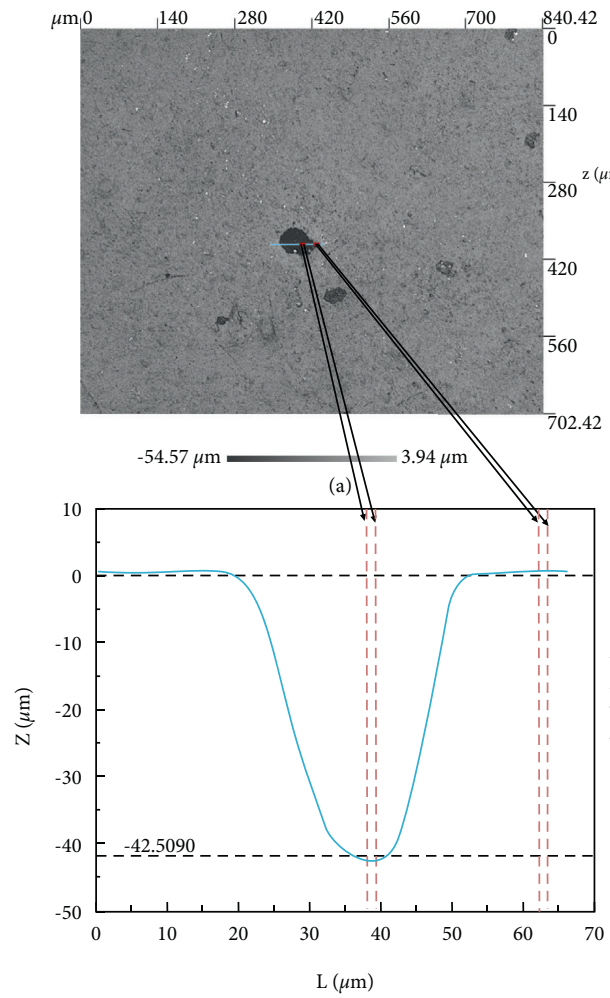

(c)
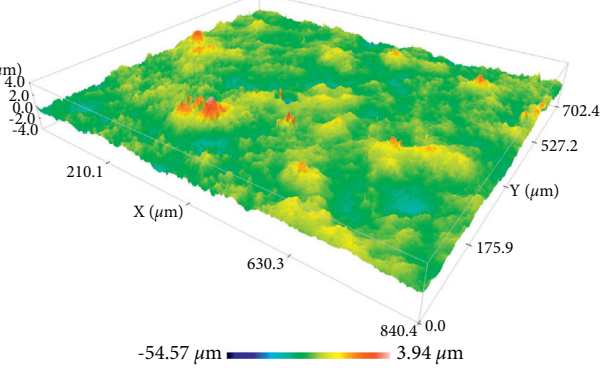

(b)

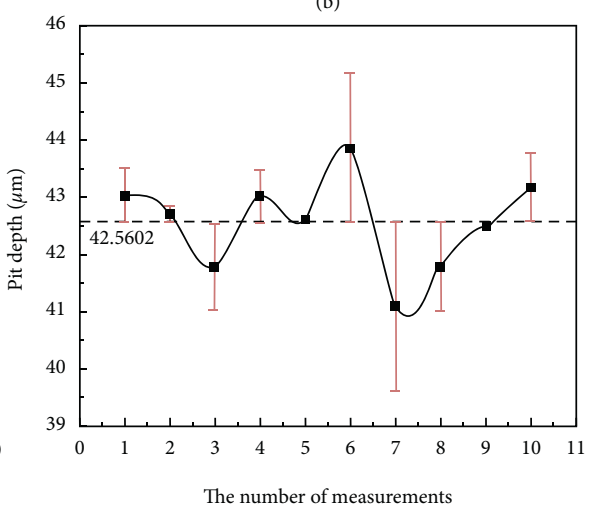

(d)

Figure 8: Measurement of the surface microscopic morphology and depth of primer after 36 laser pulses vertical action primer surface: (a) two-dimensional morphology, (b) three-dimensional morphology, (c) depth measurement, and (d) results of depth measurement. The error bars show the standard deviation of the average measurement value.

Through comprehensively analyzing the intensity of the sensitive spectral lines of the elements and the damage to the substrate, the increase of the characteristic peak intensity of $\mathrm{Ca}$ I at $422.7 \mathrm{~nm}$ can be selected as the basis for the removal of the topcoat. The decrease of the characteristic peak intensity can be selected as the basis for the removal of the primer. Figure 11 shows the changes of the LIBS signal at different pulse numbers of $\mathrm{Ca}$ I at $422.7 \mathrm{~nm}$ vertically acting 


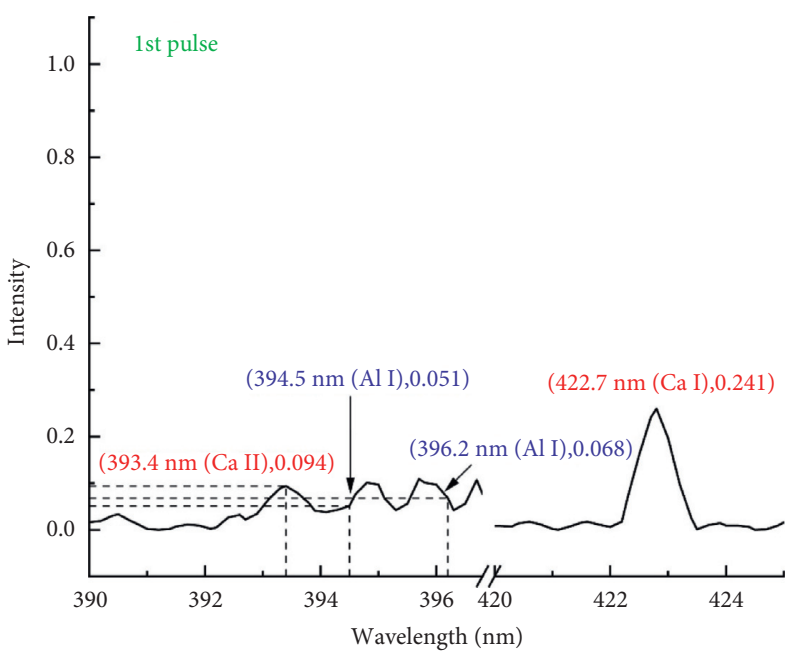

(a)

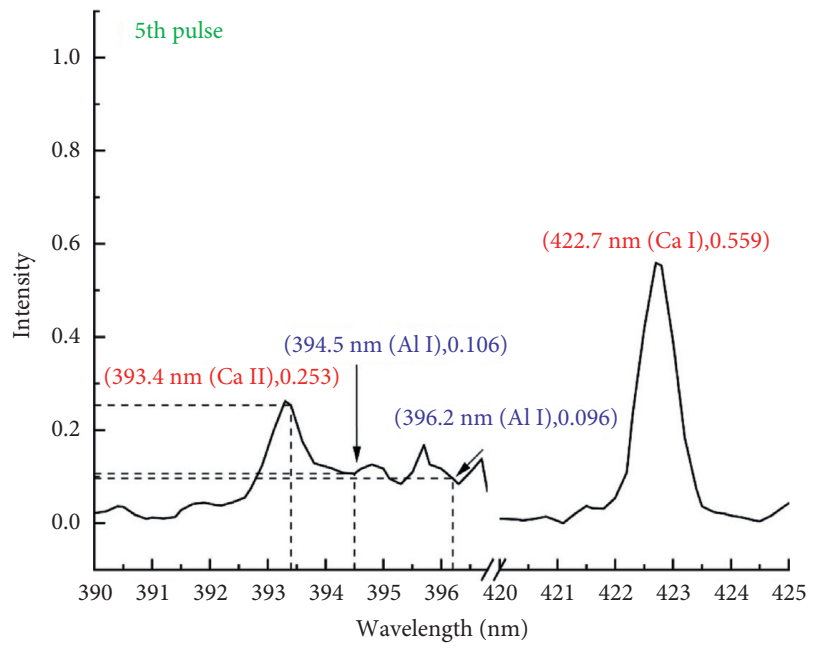

(c)

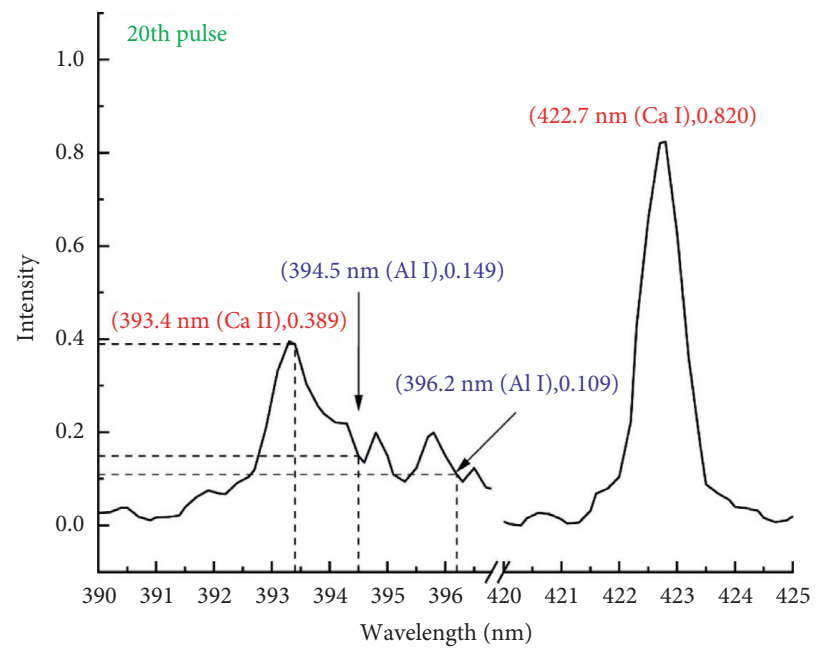

(e)

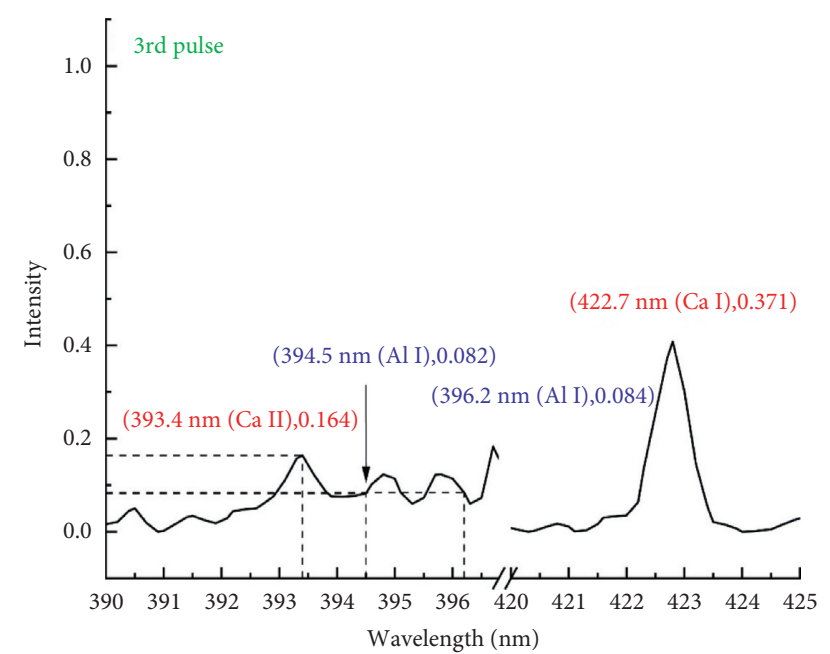

(b)

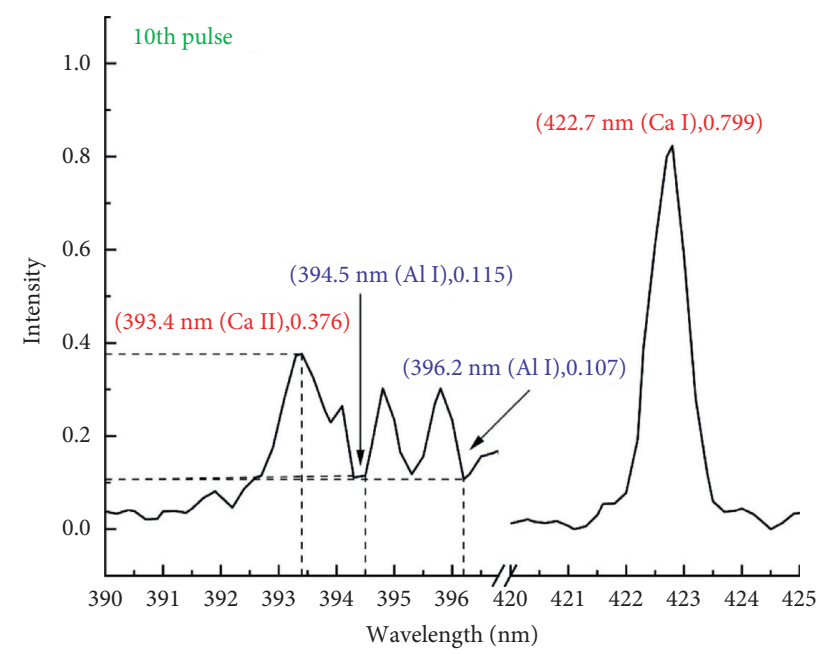

(d)

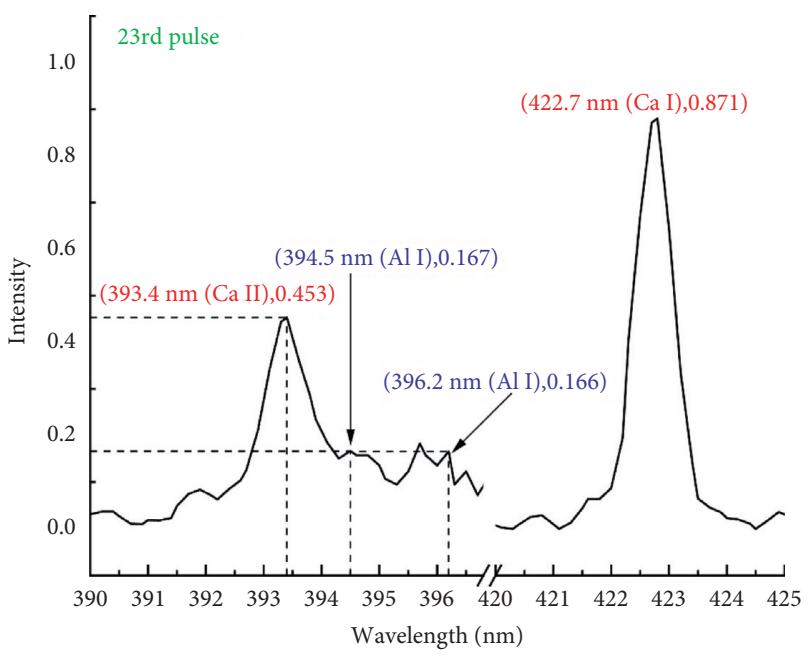

(f)

FIgURE 9: Continued. 


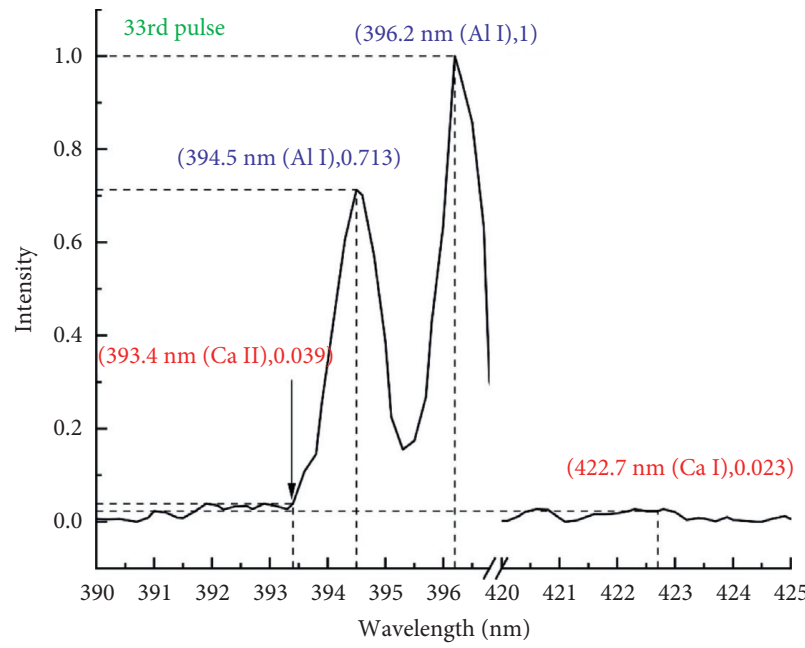

(g)

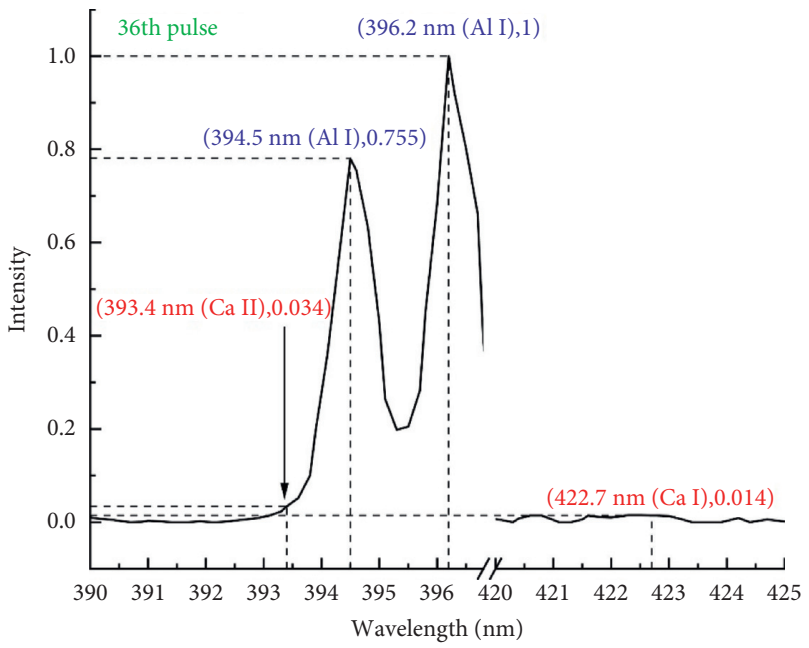

(h)

FIGURE 9: Laser-induced breakdown spectroscopy characteristic peak of primer after different times of laser pulse acting vertically on the surface: (a) 1, (b) 3, (c) 5, (d) 10, (e) 20, (f) 23, (g) 33, and (h) 36.

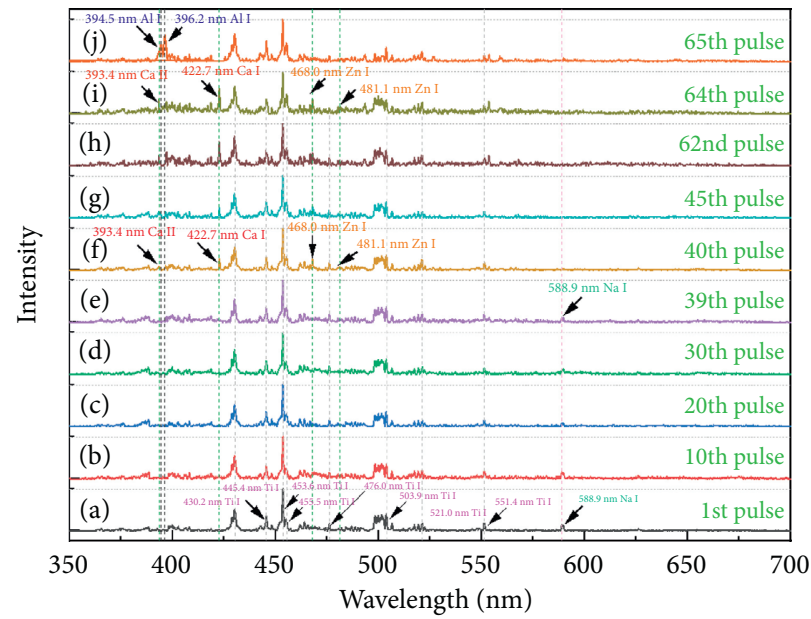

FiguRE 10: Laser-induced breakdown spectroscopy signal after different pulse numbers acting vertically on the topcoat surface: (a) 1 , (b) 10, (c) 20, (d) 30, (e) 39, (f) 40, (g) 45, (h) 62, (i) 64, and (j) 65 .

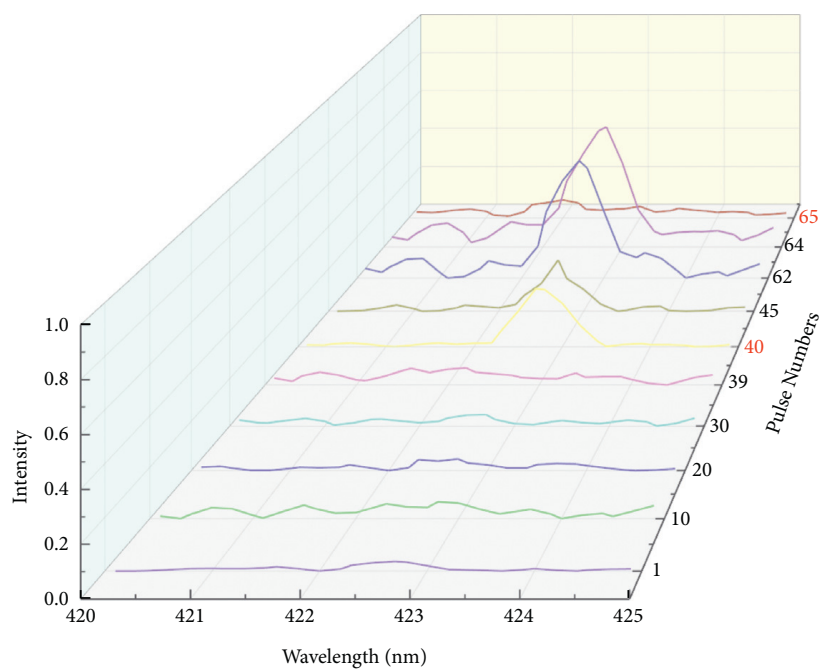

FIGURE 11: Laser-induced breakdown spectroscopy characteristic peak of Ca I at $422.7 \mathrm{~nm}$ after laser pulses of different times vertically acting on the topcoat surface. 
on the surface of the topcoat. It can be observed that the intensity increased at the 40th pulse and decreased at the 65th pulse.

\section{Conclusions}

The LIBS technology was used to measure the spectral signal changes on the surface of the aluminum alloy, primer, and topcoat at different pulse numbers. It may be concluded that the appearance of the LIBS characteristic peak of Ca I at $422.7 \mathrm{~nm}$ indicates that the topcoat was completely removed, and the disappearance of the characteristic peak indicates that the primer was completely removed during the laser paint removal process. The appearance of the LIBS characteristic peak of $\mathrm{Al} \mathrm{I}$ at $394.5 \mathrm{~nm}$ or $396.2 \mathrm{~nm}$ indicates that the laser was acting on the aluminum alloy substrate. The results in this work could prove the LIBS characteristic peak of the characteristic elements of a specific paint layer with the signal change of the laser paint removal process, the laser-based layered controlled paint removal (LLCPR) can be realized, which proves the feasibility of LIBS technology for online monitoring and feedback of laser paint removal process.

\section{Data Availability}

The data used to support the findings of this study are available from the corresponding author upon request.

\section{Conflicts of Interest}

The authors declare that there are no conflicts of interest regarding the publication of this article.

\section{Acknowledgments}

This work was financially supported by (i) Sichuan Province Science and Technology Planning Project "Research and Development of Aircraft Skin Laser Automatic Paint Removal Equipment" (2021YFSY0025), (ii) Achievement Transformation Project of Civil Aviation Flight University of China "Research on Laser Intelligent Surface Treatment Technology and Application of Aircraft Fiber Reinforced Composite Material Components" (CJ2019-03), and (iii) Postgraduate Research and Innovation Fund Project of Civil Aviation Flight University of China "Research on laser paint removal monitoring and feedback based on LIBS technology"(X2021-9), and (iv) Undergraduate Innovation and Entrepreneurship Project "Study on the Evolution Law of Plasma Spectra in the Thickness Direction of the Surface Paint of Aircraft Skin" (S202110624074).

\section{References}

[1] H. R. Sun, Research on Laser Composite Cleaning Process and Removal Mechanism of Paint Coating on Aluminum Alloy Surface, Harbin Institute of Technology, Harbin, China, 2020.

[2] J. H. Gan, "U.S. Navy MRO center tests laser cleaning technology for aircraft components," Aviation Maintenance \& Engineering, vol. 05, p. 18, 2021.

[3] S. Jin, J. X. Wang, X. D. Yuan, Y. Hu, and C. Z. Yao, "Laser paint removal technology for aircraft metal skins and composite surfaces," Aeronautical Manufacturing Technology, vol. 61, no. 17, pp. 63-70, 2018.

[4] G. X. Chen, T. J. Kwee, K. P. Tan, Y. S. Choo, and M. H. Hong, "Laser cleaning of steel for paint removal," Applied Physics A, vol. 101, no. 2, pp. 249-253, 2010.

[5] M. K. A. A. Razab, M. S. Jaafar, F. M. Suhaimi et al., "Study on performance of $\mathrm{CO}_{2}$ laser in paint removal over selected national car model," Journal Technology, vol. 78, no. 3, pp. 203-209, 2016.

[6] A. Kumar and D. J. Biswas, "Particulate size and shape effects in laser cleaning of heavy metal oxide loose contamination off clad surface," Optics \& Laser Technology, vol. 106, pp. 286-293, 2018.

[7] J. Savovic, M. Momcilovic, S. Zivkovic, A. Stancalie, M. Trtica, and M. Kuzmanovic, "LIBS analysis of geomaterials: comparative study of basalt plasma induced by TEA $\mathrm{CO}_{2}$ and Nd: YAG laser in air at atmospheric pressure," Journal of Chemistry, vol. 2017, Article ID 9513438, 9 pages, 2017.

[8] G. David, Y. Meslin, E. Dehouck et al., "Laser-induced breakdown spectroscopy (LIBS) characterization of granular soils: implications for Chem Cam analyses at gale crater, Mars," Icarus, vol. 365, Article ID 114481, 2021.

[9] L. Emmanuel, K. Menelaos, L. Elizabeth et al., "EXPRESS: combined spectroscopic analysis of terrestrial analogs from a simulated astronaut mission using the laser-induced breakdown spectroscopy (LIBS) Raman sensor: implications for Mars," Applied Spectroscopy, vol. 37028211016892, 2021.

[10] Z. Gazali, R. Kumar, K. R. Pradeep, P. Rai, A. K. Rai, and S. N. Thakur, "Discrimination of gallbladder stone employing laser-induced breakdown spectroscopy (LIBS) and photoacoustic spectroscopy (PAS)," Spectrochimica Acta Part A: Molecular and Biomolecular Spectroscopy, vol. 260, Article ID 119948, 2021.

[11] M. Aida, S. Giorgio, R. Anielle et al., "Discrimination of genetically very close accessions of sweet orange (citrus sinensis L. Osbeck) by laser-induced breakdown spectroscopy (LIBS)," Molecules, vol. 26, no. 11, Article ID 3092, 2021.

[12] Y. Zhang, T. L. Zhang, and H. Li, "Application of laser-induced breakdown spectroscopy (LIBS) in environmental monitoring," Spectrochimica Acta Part B: Atomic Spectroscopy, vol. 181, Article ID 106218, 2021.

[13] P. Silvia, F. M. L. Javier, V. Marco et al., "Chemometrics and elemental mapping by portable LIBS to identify the impact of volcanogenic and non-volcanogenic degradation sources on the mural paintings of Pompeii," Analytica Chimica Acta, vol. 1168, Article ID 338565, 2021.

[14] R. J. Jegan, C. Bin, and M. Srinivasan, "A review on the recycling of spent lithium-ion batteries (LIBs) by the bioleaching approach," Chemosphere, vol. 282, Article ID 130944, 2021.

[15] H. M. Abir, P. K. Diwakar, and R. Shankarachary, "Total nitrogen estimation in agricultural soils via aerial multispectral imaging and LIBS," Scientific Reports, vol. 11, no. 1, Article ID 12693, 2021.

[16] R. Ledesma, F. Palmieri, and J. Connell, "Laser induced breakdown spectroscopy of polymer matrix composites for real-time analysis of trace surface contaminants: a review," International Journal of Adhesion and Adhesives, vol. 98, Article ID 102528, 2020.

[17] X. D. Wang, J. Yu, Z. Q. Mo, X. Jianguo, D. Shoujun, and M. Jingjing, "Research progress of laser paint removal technology," Laser \& Optoelectronics Progress, vol. 57, no. 5, pp. 59-70, 2020. 
[18] X. Li and Y. Guan, "Real-time monitoring of laser cleaning for hot-rolled stainless steel by laser-induced breakdown spectroscopy," Metals, vol. 11, no. 5, Article ID 11050790, 2021.

[19] L. X. Sun, W. J. Wang, L. F. Qi, L. Ying, Q. Hongchao, and W. Wei, "Online monitoring of laser cleaning effect of carbon fiber composites based on laser-induced breakdown spectroscopy technology," Chinese Journal of Lasers, vol. 47, no. 11, pp. 299-308, 2020.

[20] Y. Tong, X. Chen, A. Zhang et al., "Research on laser cleaning plasma spectroscopy online detection of carbon fiber composite materials," Spectroscopy and Spectral Analysis, vol. 39, no. 8, pp. 2388-2394, 2019.

[21] Y. Q. Tong, Q. H. Lu, and J. Z. Zhou, "On-line detection of plasma spectroscopy for laser cleaning of Aluminum alloy before welding," Spectroscopy and Spectral Analysis, vol. 40, no. 1, pp. 255-260, 2020.

[22] K. X. Chen, G. Y. Feng, G. L. Deng, C. Liu, and D. Wang, "Study on the mechanism of laser paint removal based on emission spectrum and composition analysis," Spectroscopy and Spectral Analysis, vol. 36, no. 9, pp. 2956-2960, 2016.

[23] L. Chen, G. L. Deng, G. Y. Feng, X. H. Yan, and J. Q. Li, "Study on the mechanism of laser paint removal based on LIBS and time-resolved characteristic peaks," Spectroscopy and Spectral Analysis, vol. 38, no. 2, pp. 367-371, 2018.

[24] Q. Zhou, G. Deng, Y. Chen, and S. Zhou, "Laser paint removal monitoring based on time-resolved spectroscopy," Applied Optics, vol. 58, no. 34, pp. 9421-9425, 2019.

[25] V. Lazic and A. De Ninno, "Calibration approach for extremely variable laser induced plasmas and a strategy to reduce the matrix effect in general," Spectrochimica Acta Part B: Atomic Spectroscopy, vol. 137, pp. 28-38, 2017.

[26] R. Noll, Laser-Induced Breakdown Spectroscopy, Springer, Berlin, Germany, pp. 7-15, 2012. 